\title{
A Review on Dietary Intervention in Obesity Associated Colon Cancer
}

\author{
N H Roslan, S Makpol, Y A Mohd Yusof*
}

\begin{abstract}
Background: Colorectal cancer (CRC) is one of the major causes of morbidity and mortality. According to National Cancer Registry, the incidence of colorectal cancer in Peninsular Malaysia increases with age. The incidence is highest among Chinese population but lower among Indians and Malays. Many reviews have suggested that obesity may be associated with a higher risk $(>50 \%)$ of colorectal cancer. Methods: This study collects a comprehensive data from the literature review available from respective journals on dietary intervention and the chemo-protective mechanisms of a few natural resources in obesity -associated colon cancer based on previous and current studies. Results: In obesity-associated colon cancer, the genes of interest and pathways that are mainly involved include NFkB, P13K/Akt, and MAPK pathways, and FTO, leptin, Cyclin D, MMPs, and STAT3 genes. Dietary modification is one of the alternative steps in early prevention of colon cancer. It has been proposed that the components present in certain foods may have the ability to protect against many diseases including the prevention of cancer. Conclusion: There are many factors that lead to obesity-associated colon cancer and the mechanisms behind it is still undergoing intensive research. This review aims to scrutinize research as well as reviews that have been previously reported on obesity associated colorectal cancer and the beneficial effects of including antioxidants-rich foods such as vegetables and fruits in the diet to reduce the risk of obesity associated colorectal cancer.
\end{abstract}

Keywords: Obesity- colon cancer- pathways- natural phytochemicals

Asian Pac J Cancer Prev, 20 (5), 1309-1319

\section{Introduction}

Colorectal cancer (CRC) is one of the main factors of mortality and morbidity. According to World Health Association (WHO), cancer is a cause of death worldwide, accounting for 8.2 million deaths in 2012. WHO also reported that colorectal cancer is the third most common cancer in men, (746,000 cases, $10.0 \%$ of the total) and the second in women, $(614,000$ cases, $9.2 \%$ of the total $)$ around the world (WHO, 2012). It is expected that annual cancer cases will slowly increase from 14 million in 2012 to 22 million within the next 2 decades. Colorectal cancer is the most common cancer among male and second among female in Peninsular Malaysia. The incidence of colorectal cancer is expected to increase with age and is highest among Chinese population but is lower among Indian and Malay population according to the National Cancer Registry 2006 (National Cancer Registry, 2006). Obesity is known to be the major global health problem that can lead to co-morbidities including diabetes, heart disease, and cancer (Calle et al., 2003; Drew, 2012; Percik and Stumyoll, 2009; Wollin et al., 2010). The Star Online (2014) stated that $45.3 \%$ of Malaysian population is obese followed by South Korea with 33.2\%, Pakistan 30.7\% and China, 38.3\% making Malaysia with the most obese population in South East Asia. It has been reported that there is a link between colorectal cancer and obesity in comparison to other type of cancers (Monghaddam et al., 2007). The WHO 2015 Fact Sheet reported that over 600 million people worldwide were obese (WHO, 2015). In the US, NHANES (National Health and Nutrition Examination Survey) reported that between 2011-2014, more than one third of adults (36.5\%) aged 20 years or older are obese (National Centre for Health Statistics, 2015). While in the Asia Pacific Region, Malaysia has the highest rate of obesity (14\%) followed by Thailand $(8.8 \%)$, and Vietnam and India have the lowest rates of obesity (1.7\% and $1.9 \%$ respectively (WHO, 2015; National Health and Morbidity Survey, 2006).

It is estimated that obesity is responsible for about $14 \%$ of cancer caused-death (Calle et al., 2003). In a meta-analysis study, it was observed that general and central obesity was positively associated with the risk of colorectal cancer (Monghaddam et al., 2007). In obesity associated colon cancer, multiple signalling pathways have been reported to be involved with some genes of interest for example the inflammation pathway $\mathrm{NF \kappa B}$, survival pathways P13K/Akt and MAPK, and FTO, leptin, Cyclin 
D, MMPs, and STAT3 genes (Chen et al., 2013). One of the alternative ways for primary prevention of cancer is by dietary modification. It has been proposed that the components present in certain foods may have the ability to protect against many diseases which has become the field of interest for researchers in recent years (Sunkara et al., 2009).

\section{Risk Factors of Colorectal Cancer}

The pathogenesis of colorectal cancer is very intricate and is influenced by several factors. There are several risk factors associated with the incidence of colorectal cancer. Those that cannot be controlled by an individual, include age and hereditary factors (Haggar and Boushey, 2009).

\section{Family History and Inherited Genetic Risk}

Other than age, family history also plays a crucial role in the risk of developing colorectal cancer, such as personal history of adenomatous polyps, inflammatory bowel disease, and inherited genetic disorder (Haggar and Boushey, 2009). Detection and removal of a polyp prior to malignancy may lower the chance of developing colorectal cancer (Grande et al., 2008). However, full removal of adenomatous polyp or localized carcinoma has been implicated in increasing development of metachronous cancer elsewhere in the colon and rectum (de Jong et al., 2005).

Adenomatous polyposis C (APC) is a tumour suppressor protein, and mutation of APC gene is the initial factor in colon carcinogenesis followed by KRAS and TP53 mutations (Arends, 2000). APC is mutated in $95 \%$ of familial adenomatous polyposis (FAP). APC is known to bind to $\beta$-catenin leading to ubiquitin-mediated beta catenin destruction as well as loss of APC function and increasing transcription of $\beta$-catenin targets. Together with the interaction with transcription factor $\beta$-catenin, APC gene product indirectly regulates transcription of some critical cell proliferation genes including cyclin D, C-myc, ephrins, and caspases (Sameer, 2013).

\section{Diet and Physical Activity}

High fat diet, especially animal fat, could lead to formation of colorectal cancer. Several studies proposed that people who consume an unbalanced diet and diet low in fruits and vegetables may have an increased risk of colorectal cancer (Janout and Kollárová, 2001).

Other than diet, two interrelated and modifiable risk factors are the excess body weight and physical inactivity. Obesity or extra body weight has been associated with colorectal cancer (de Jong et al., 2005). There is enough evidence showing that increased level of physical activity is associated with a reduce risk of colorectal cancer and this includes evidence of a dose response effect with frequency and intensity of exercises inversely associated with the risk (de Jong et al., 2005; Zisman et al., 2006; Boyle and Langman, 2000). In addition to frequent exercises, a healthy diet lifestyle can help reduce the risk of colon cancer. Thus, lack of daily physical activity could lead to a higher incidence of obesity in both women and men.

\section{Gut microbiome}

Microbiomes that live in the human gastrointestinal tract (collectively known as the gut microbiota, or microbiome) in obese individuals are different from non-obese people. Studies in mouse models suggest two theories that are highly associated with colorectal cancer, the first theory is regarding the imbalances in the gut microbiota or a dysbiotic microbial community in the gut with pro-carcinogenic features driving towards proinflammatory state leading to inflammation of epithelial cells, altered metabolism, and genotoxicity. The second theory or the "driver-passenger" theory, indicates that the intestinal bacteria as "bacteria drivers" initiate colorectal cancer by inducing epithelial DNA damage leading to carcinogenesis (de Jong et al., 2005).

\section{Pathways in CRC}

The pathogenesis of CRC stems from either genetic or epigenetic changes that are associated to each other at different degrees. The major cause of many CRC is The Chromosomal Instability (CIN) pathway indicated by widespread loss of heterozygosity $(\mathrm{LOH})$ and gross chromosomal abnormalities (Leary et al., 2008; Lin et al., 2003). Another cause of CRC is derangement of the DNA Mismatch Repair (MMR) system leading to a failure in the proofreading system of DNA polymerase.

\section{Chromosomal Instability Pathway (CIN Pathway)}

The chromosomal instability (CIN) is one of the pathways that features the genomic instability acquired during tumour development in the pathogenesis of colorectal cancer. It is characterised by widespread imbalances in chromosome number (aneuploidy) and loss of heterozygosity (LOH) (Pino and Chung 2011).

\section{The Wnt Signalling Pathway}

The main or initial mutation in CIN pathway is the

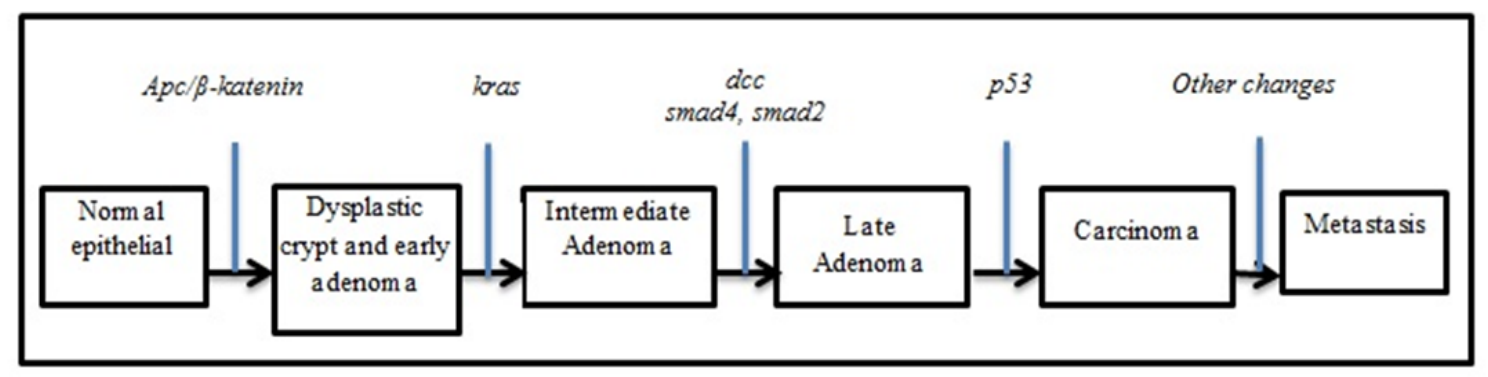

Figure 1. Morphological Change in Colon Carcinogenesis. (Adapted from: Van de Flier et al., 2007). 


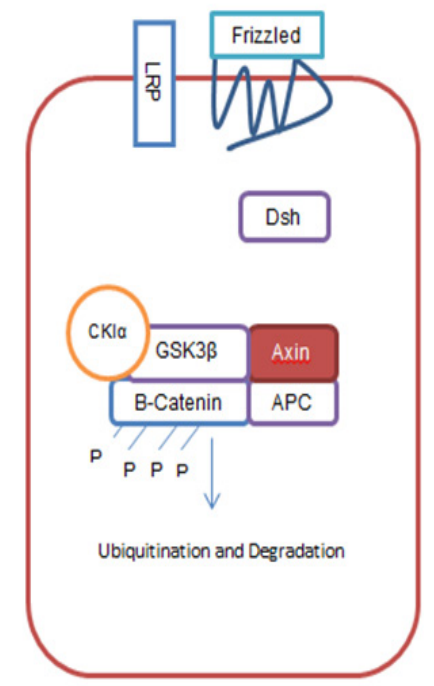

Absence of Wnt Ligand

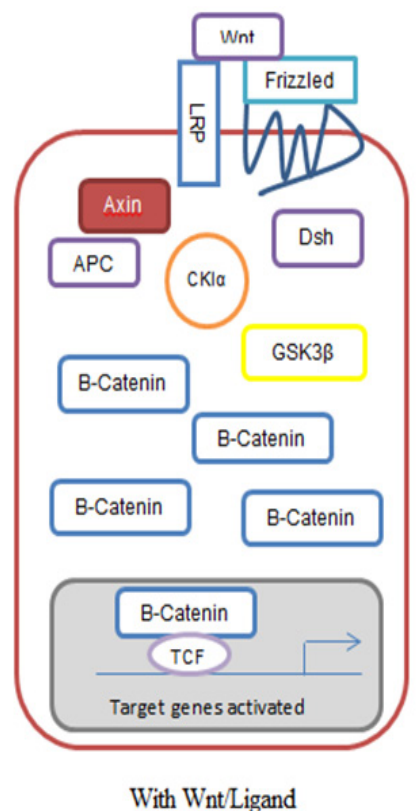

Figure 2. A Simplified Version of the Canonical Wnt/ Beta-Catenin Pathway. Absence of Wnt/Ligand (2A); With Wnt/ Ligand (2B). (Adapted from: Van de Flier et al., 2007)

early mutation on tumour suppressor gene, adenomatous polyposis coli (APC) involving sporadic CIN and also when there is a mutation in the germline, in Familial Adenomatous Polyposis (FAP) (Shih et al., 2001; Sieber et al., 2012). Wnt signalling pathway controls the level of modulator beta catenin for signal transduction via phosphorylation processes and ubiquitin-mediated degradation. APC protein binds to beta catenin and undergoes ubiquitination; essentially as a negative regulator of beta catenin (Novellasdemunt et al., 2015). The loss function of APC either through mutation, $\mathrm{LOH}$, or promoter methylation, will result in constitutive activation of Wnt signalling, thus, accumulation of cytoplasmic beta catenin (Novellasdemunt et al., 2015). This results in nuclear translocation and binding of beta catenin to T-cell factor (TCF)/ lymphoid enhancer factor (LEF) with increased proliferation, differentiation, migration and adhesion of colorectal cells. A simplified diagram of the Wnt/ beta-catenin pathway is presented in Figure 2. The Wnt target genes such as cyclin D and c-myc-c affect various cellular functions including cell proliferation, angiogenesis, and apoptosis. Mutation of Wnt target genes will cause degeneration of these cellular functions leading to both initiation and progression of CRC.

\section{RAS Pathway}

The progression of adenoma to carcinoma is identified initially by the K-ras gene, a proto-oncogene which

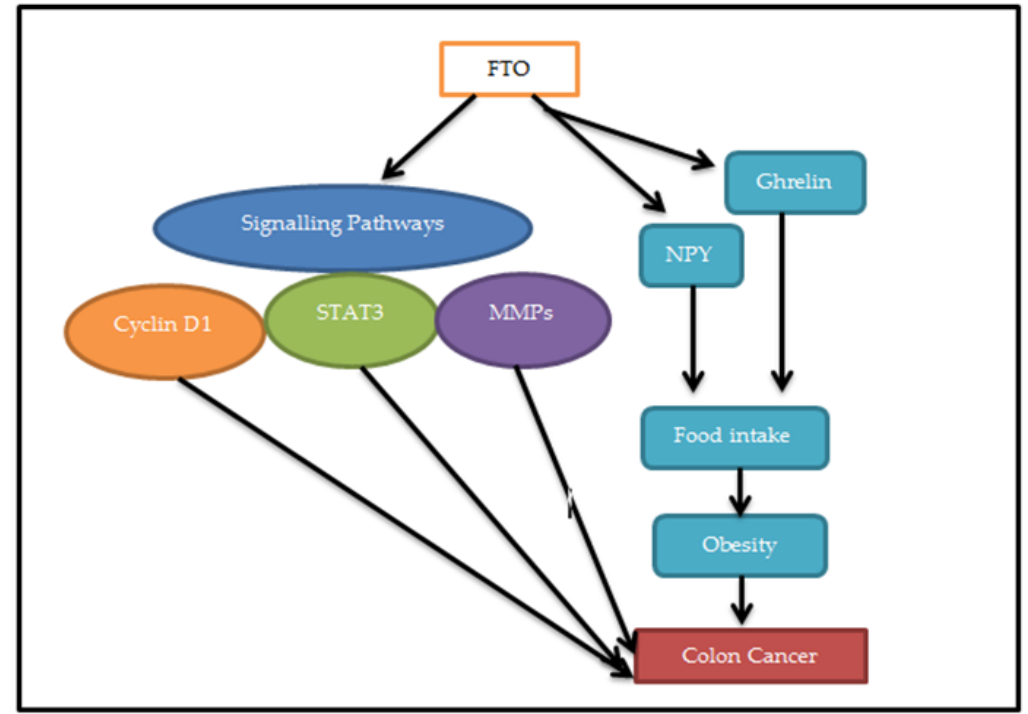

Figure 3. The Links between FTO and Colon Cancer. Over-expression of FTO protein could increase incidence of colon cancer through both obesity dependent and independent pathways. FTO increases food intake through satiety hormone leptin, causing obesity. In independent obesity associated pathway, FTO can initiate cellular signalling molecules STAT3, cyclin D1 and MMPs to increase cancer incidence. FTO, Fat mass and obesity associated; STAT3, signal transducer and activator of transcription 3; MMPs, matrix metalloproteinase; NPY, neuropeptide Y. (Adapted from Chen et al., 2013) 


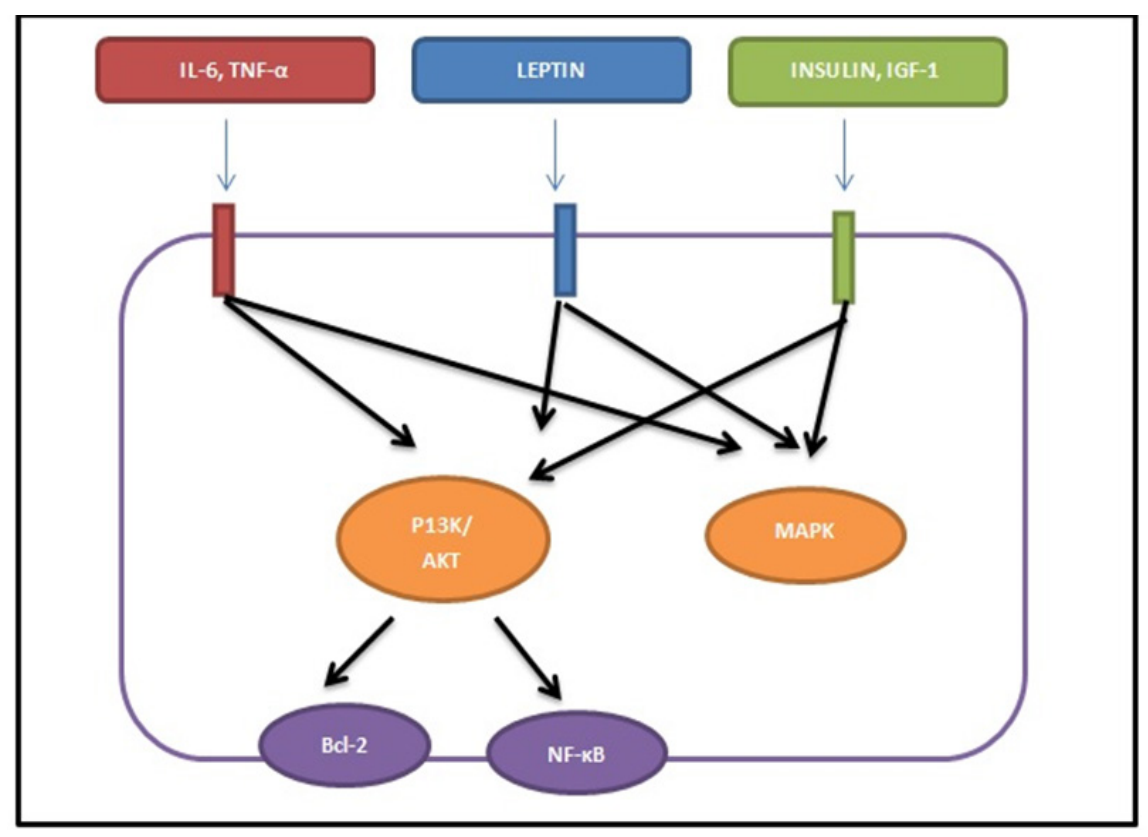

Figure 4. Signal Pathways in Obesity Associated Colon Cancer. Phosphorylation of receptors by the ligands such as IL-6, Leptin and IGF-1 leads to activation of downstream pathways PI3K/AKT and MAPK. Abbreviation: IGF-1; Insulin like growth factor-1; IL6, interleukin 6; PI3K/Akt, phosphoinositide 3-kinase/protein kinase B; MAPK, mitogen activated protein kinase; NF- $\mathrm{B}$, nuclear factor-kappa; B bcl-2, B-cell lymphoma 2 (Adapted from Chen, 2011).

coded for the GTP-ase protein that takes place in the transduction and propagation of extracellular signals such as mitogen-activated protein kinase (MAPKs). K-ras mutation leads to an irreversible active state that allows the cell to escape apoptosis but accelerates in proliferation. More than $90 \%$ of K-ras gene mutations occur at codon 12 and 13; where codon 12 mutation denotes an oncogenic phenotype compared to mutation at codon 13 (Malumbres and Barbacid, 2003). This implies mutations on codon 13 are towards the adenoma-carcinoma transition; whereby codon 12 mutations predispose colorectal tumour cells to surrounding invasion and metastasis (Guerrero, 2000). Horst and colleagues proved that increased activity of WNT was coherent with elevated MAPK signalling, in association with CRC K-ras mutated event (Horst et al., 2012). The ERK-MAPK pathway is found downstream of several growth-factor receptors, including epidermal growth factor, EGF-R (Horst et al., 2012). Uncontrolled expression and activation of EGF-R coupled with uncontrolled expression and activation of ERK- MAPK pathway are prerequisite in colorectal cancer (Baba et al., 2010).

\section{The P53 System}

The loss of function of p53 generally occurs in the progressive and later stages of colorectal carcinogenesis. The mutation of p53 gene triggers an increase in proliferative activity via the loss of apoptosis and cell cycle control (Boyle and Langman, 2000). A study conducted by Oikawa, showed that p53 is largely controlled by BubR1 which is a component of the mitotic spindle checkpoint machinery. Loss of Heterozygosity $(\mathrm{LOH})$ at $17 \mathrm{p}$ significantly downregulates the activity of BubR1 (Oikawa et al., 2005). El-Deiry and coworkers established that a wild-type p53-activated fragment 1
(WAF-1), a highly induced gene has the ability to suppress growth of tumour cells growth (el-Deiry et al., 1993). However, when p53 is mutated, the protective role of WAF-1 is lost. During the advancement of carcinogenesis, mutations in several cyclin-dependent kinases (CDKs) are also involved. Through the AMPK pathway, p53 up-regulates the CDK inhibitor 1A (CDKN1A or p21), involved in cell cycle regulation. Another CDK related with p53 mutations is CDK inhibitor 1B (CDKN1B or p27). Mutated P53 cross talks with Cyclooxygenase-2 (COX-2), initiating cell proliferation and inflammation in CRC (Swamy, 2003).

\section{Microsatellites (MSI) Pathway}

Microsatellites are short repeated nucleotide sequences which are extended to the whole genome. These are susceptible to errors throughout replication caused by their repetitive manner. The DNA mismatch repair (MMR) system identifies and then repairs base-pair mismatches that takes place during replication of DNA. The instability of microsatellites reflects on the incapacity of the MMR system to repair these problems. These problems are then identified through frameshift mutations in the microsatellite repeats (Dora et al., 2013). It was found that there is a link between MSI with HNPCC (Hereditary Nonpolyposis Colorectal Cancer). HNPCC is the results of germline mutation in MMR gene, whereas somatic hypermethylation or mutation silencing of MMR genes involves about $15 \%$ of sporadic CRC. Members of the MMR system include Mut S homologue 2 (MSH2), Mut L homologue 1(MLH1), Mut S homologue 6 (MSH6), post-meiotic segregation-1 (PMS1), Mut L homologue 3(MLH3), Mut S homologue 3 (MSH3), and exonuclease 1(Exo1) (Dora et al., 2013). 
CIMP (Cpg Island Methylator Phenotype) and the "Serrated" Pathway

Changes in the expression or function of gene without modifying sequence of the DNA of that certain gene are known as epigenetic alterations. In humans, epigenetic changes are commonly either due to modifications of histone or DNA methylation (Samowitz et al., 2005). DNA methylation happens frequently at the $5^{\prime}-\mathrm{CG}-3^{\prime}(\mathrm{CpG})$ dinucleotide. Gene promoter region methylation results in gene silencing, resulting in loss of function of tumour suppressor genes (Ogino et al., 2006). CIMP accounts to the presence of hypermethylation of numerous genes. The genes which are silenced via hypermethylation of DNA during colon carcionogenesis include MCC (the mutated in colorectal cancer) gene, APC (adenomatous polyposis coli), MGMT (O6-methylguanine DNA methyltransferase), and MLH1. Environmental factors such as age and excessive smoking have been shown to escalate DNA methylation (Dora et al., 2013; Ogino et al., 2006). Five markers have been selected to act as markers for CIMP which consists of CACNA1G (Calcium Channel, Voltage-Dependent, T Type, Alpha 1G Subunit), IGF2 (insulin-like growth factor 2), NEUROG1 (Neurogenin 1), RUNX3 (Runt-Related Transcription Factor 3), and SOCS1 (Suppressor of cytokine signaling 1). CIMP positivity is known as methylation on at least three markers (Dora et al., 2013). Pathologically, CIMP-high tumours are usually poorly differentiated of signet ring or mucinous histology, microsatellite instability, and harbor mutation of BRAF.

\section{Multiple Signal Pathways in Obesity Associated Colon Cancer}

Various epidemiological studies have proven that obesity enhances the incidence of colon cancer (Ben et al., 2012; Pischon et al., 2006). In a study conducted by Pischon and co-workers, they showed that the body mass index (BMI) and body weight were significantly correlated to colon cancer risk in men, while weaker or zero associations have been reported for women (Pischon et al., 2006). However, the reason for the apparent discrepancy in the relation between body weight with colon cancer risk between women and men are still debatable and one of the possible reasons is due to the different in body composition between women and men (Pischon et al., 2006).

To prevent obesity-associated colon cancer, it is of extremely importance to recognize the shared molecular mechanisms involved in colon cancer and obesity as there are multiple signalling pathways shown to be associated with obesity-associated cancers (Chen et al., 2013; Chen, 2011). In addition, it is uncertain whether there is shared genetic bias between obesity and cancers. Until now, 30 gene loci have been reported (Chen, 2012). Some example of the genes that are activated in obesity associated colon cancer include, insulin, insulin-like growth factor, estrogen, leptin, adinopectin, interleukin-6 (IL-6), IL-17, tumour necrosis factor alpa (TNF- $\alpha$ ) (Chen, 2012; Flores et al, 2012; Gislette and Chen, 2010; Paz-Filho et al., 2011; Sikilidis and Varamini, 2001). In addition, genes of the MAPK, P13/K and beta-catenin pathways were also reported to be initiated in obesity associated colon cancer. Some other genes of interest include FTO, leptin, Cyclin D, MMPs and STAT3 (Arends, 2000). Therefore, inhibition of these factors and pathways involved offer useful knowledge in the prevention of obesity associated colon cancer.

\section{The PI3K/Akt Pathway}

In $\mathrm{P} 13 \mathrm{~K} / \mathrm{Akt}$ pathway, PI3K converts PIP2 (Phosphatidylinositol $(3,4,5)$-trisphosphate) to PIP3 (Phosphatidylinositol (4,5)-trisphosphate), which then activates putative 3-phosphoinositide-dependent kinase 1 (PDK1) thus phosphorylating Thr308 of Akt driving to its activation. In order to further increase the activity of the kinase, mammalian target of rapamycin complex 1 (MTORC2) phosphorylates another moiety (Ser473) of Akt. Activated Akt can boost carcinogenesis including obesity-induced colon cancer through multiple targets such as blocking check point kinase to increase genomic instability; increased mTORC1 (mammalian target of rapamycin) activity to promote protein synthesis; increased cell cycling through glycogen synthase kinase 3b (GSK3b) inhibition; blocking MDM2 (murine double minute) to decrease p53; increased NF-kB leading to increased survival; and activating HIF1-a (Hypoxiainducible factor 1-alpha) to increase expression of vascular endothelial growth factor (VEGF) for angiogenesis (Chen, 2011).

\section{Mitogen-Activated Protein Kinase (MAPK) Signal Pathway}

MAPK pathway consists of Ras-Raf-MEKERK1/2 pathways. Ras phosphorylates Raf which then phosphorylates MEK and the latter will phosphorylate ERK1/2. ERK is also known to activate STAT3 and mTOR (Grande et al., 2008). Other pathways involved are the p38 MAPK and JNK (c-Jun N-terminal kinases) pathways. The cross talk between these pathways result in cancer progression and metastasis (Huang et al., 2011). STAT3 controls many genes for example, IL-17, IL-23, B-cell lymphoma 2 (Bcl-2), Bcl-xL, Mcl1, cyclin D1 and vascular endothelial growth factor to increase cell survival, proliferation, invasion, angiogenesis and metastasis (Aggarwal et al., 2009; Yu et al., 2009). An epidemiological study reported that high IGF-1 and insulin levels correspond with obesity and waist circumference, increasing the risk of colon cancer.

Nunez et al., (2009) proposed the link between insulin/ IGF pathway and obesity-associated cancer. Insulin together with activated $\mathrm{mTOR}$ has been proposed to be an important reason behind the elevation of cancer occurrence and mTOR suppression via rapamycin destroyed high cancer incidence in diabetic patient (Fierz et al., 2010; Novosyadlyy et al., 2010). In mouse models, reduced levels of IGF-1 either through genetic modification or energy restriction lowered skin cancer progression by suppression of PI3K/Akt/mTOR pathway (Moore et al, 2008). IGF-1 genetically deficient mice, has been associated with decreased azoxymethane-induced colon cancer indicating that decreased in circulating IGF-1 levels possibly block colon carcinogenesis and affect proliferation and apoptosis (Olivo-Marston et al., 2009). 


\section{Adipokine Leptin, Adiponectin and Cytokine}

Obesity is also implicated by decreased serum adiponectin and increased leptin, the hormones may play a role as potential mediators in obesity associated carcinogenesis (Janice, 2014). Adipokine leptin is a regulator of satiety and energy balance. It is elevated in obesity and may induce various cancers of prostate, thyroid, colorectal, renal, pancreatic, oesophageal and endometrial (Chen, 2011). Leptin is also known to modulate absorption of nutrients in gastrointestinal tract, promoting cellular growth and proliferation, inhibiting apoptosis, and stimulating pro-inflammatory responses in small and large intestine (Riondino et al, 2014). In colon cancer, leptin acts as a potent mitogen and anti-apoptotic cytokine and promotes the invasiveness of familial adenomatous colonic cells. Accordingly, leptin expression has been reported to be increased as carcinogenesis progresses from normal colonic mucosa to adenoma and adenocarcinoma. Therefore, this suggests the involvement of leptin in multistep colon carcinogenesis (Riondino et al, 2014). At the molecular level, leptin mediates its growth and proliferative effects via obesity receptor $(\mathrm{Ob}-\mathrm{R})$ to activate survival pathways such as JAK/STAT, PI3K/ Akt, and MAPK. Gao and co-workers (2012) reported that in endometrial cancer cells, leptin activated MAPK, PI3K and STAT3 pathways (Gao et al, 2012). In another study it was reported that insertion of leptin into the culture medium activated PI3K and MAPK pathways via initiation of downstream targets RhoA, Cdc42 and Rac1 (Jaffe and Schwartz 2008). These modifications of signal pathways were demonstrated to escalate the progression and cancer cells invasion.

Adiponectin is also known to be an anti-inflammatory adipokine. Blood level of adiponectin is reduced in obesity, thus increasing risk factors associated with colon cancer (Fenton and Birmingham, 2010; Saxena et al., 2010). Adiponectin plays and important role of inhibiting secretion of IL-6 through suppression of NF-kB in APCmin/+ colon cancer cells thus preventing activation of STAT3 pathway inhibiting proliferation of MC38, colon cancer cell lines (Fenton et al., 2008; Fenton and Birmingham, 2010). Increased IL-6 expression, a pro-inflammatory cytokine, has been related to advanced stage and decreased survival in CRC patients (Waldner et al 2012). IL-6 can attach to IL-6 receptor in the target cells membrane or its soluble receptor (causing trans-signalling) for activation of multiple signal pathways such as STAT3 and JAKs (Waldner et al. و 2012).

\section{Fat Mass and Obesity Associated (FTO) pathway}

FTO genotype is commonly linked to fat mass in human bodies (Sonestedt et al., 2011). FTO protein is mostly found in the hypothalamus, which indicates its possible role in satiety (Olszewski et al., 2009). Genetic variants at FTO locus has been shown to be related to satiety (Dougkas et al., 2013). Previous studies have reported that FTO affects the intake of food rather than the energy expenditure (Cecil et al., 2008; Speakman et al., 2008; Timpson et al., 2008). Another epidemiology study reported by Yang et al., (2012), demonstrated that FTO affects both average of body mass index (BMI) and variability of BMI (Yang et al., 2012). Therefore, BMI can be linked with high cancer incidence in patients with FTO SNPs. The expression of FTO was also studied in mouse model and interestingly, knock-out of FTO gene led to lean phenotype, whereas over-expression of FTO in mice increased food intake and directly results in obesity (Davies et al., 2013). Further findings suggest that copy number variants at the FTO locus could be associated with extremes of adiposity (Davies et al., 2013). Mutation of FTO gene has also been reported to raise blood levels of leptin, a known mediator for causing obesity and colon cancer incidence. Leptin is known to activate several pathways linking to colon cancer (Labayen et al., 2011).

In non-obesity associated pathway, FTO is shown to be involved in the regulation of cell proliferation. In endometrial cell lines, knock-out of FTO resulted in decreased cyclin D1 and thus reduced cell cycle and cell proliferation (Zhang et al., 2012) suggesting a strong evidence that FTO may be involved in cell proliferation directly. Over-expression of FTO in rats promotes STAT3 mRNA expression in the arcuate nucleus of the hypothalamus (Pitman et al., 2012). FTO also activates other cellular signalling molecules such as cyclin D1 and MMPs known to play a role in colon carcinogenesis. Silencing of siRNA FTO caused an increase of pAkt and decreased pAMPK, a negative regulator (Pitman et al., 2012).

The overall simplified pathways are shown in Figures 3 and 4 showing the possible links between FTO and colon cancer. Over-expression of FTO protein leads to leptin-induced obesity could increase the incidence of colon cancer via both obesity dependent and independent pathways. Phosphorylation of IGF-IR (Figure 4) recruits the downstream signalling protein insulin receptor substrate (IRS) to the cell membrane that further activates both phosphoinositide 3-kinase (PI3K)-Akt and the mitogen-activated protein kinase (MAPK) pathways. There is ample evidence from some studies to suggest that insulin and insulin-like growth factor (IGF) are involved in obesity associated colorectal cancer (Olivo-Marston et al., 2009; Khandekar et al., 2011).

\section{Colon Cancer Prevention}

Phytochemicals found in natural food compounds are ideal for chemoprevention., They are polyphenols known to possess antioxidants activity. Many epidemiological studies have demonstrated that frequent consumption of natural antioxidants found in diet is related with a lower risk of cancer (Aggarwal et al., 2009). Phytochemicals such as lycopene from tomatoes, genistein from soy products and resveratrol from grapes have the ability to lower both morbidity and mortality of many types of cancer. Other foods and herbs that have exhibited anticancer activity include garlic, soybeans, cabbage, broccoli, carrots, celery, parsley, ginger, onions, flax, turmeric, tomatoes, peppers, brown rice, wheat and honey (Sunkara et al., 2009).

Another important aspect is the understanding of the mechanisms of action of chemopreventive agents in reducing tumours. It is important to identify potential agents against colon cancer. As reported, there are 
specific constituents of foods that help shielding against cancer via several mechanisms such as antioxidant activity and trapping of oxygen radicals, induction of drug metabolizing and detoxifying enzymes, signal transduction modulation, cell proliferation and apoptosis (Sunkara et al., 2009; Aggarwal and Shishodia, 2006).

\section{Green Tea (Epigallocatechin Gallate (EGCG)}

Green tea contains strong antioxidants activity. The main constituent found in green tea is the catechin epigallocatechin gallate (EGCG). The cancer chemoprevention effect of green tea is associated with its antioxidant activities (Kumar et al., 2010; Mak, 2012). A synergistic anti-proliferative effect on colorectal cancer cell lines HCT116 and SW480 was seen when panaxadiol, a purified ginseng compound, and EGCC was combined. (Du et al., 2012; Nakazato et al., 2005).

The chemopreventive effect of green tea extracts and EGCG in colon cancers has been studied in both Apcmin/- and AOM animal models. Green tea extracts alone and in combination with sulindac was shown to reduce development of intestinal tumour by downregulation of beta-catenin in Apcmin/- mouse model (Orner et al., 2002; Suganuma et al., 2001). Ju et al., (2005) reported that oral consumption of green tea inhibited the azoxymethane (AOM)-induced aberrant crypt foci (ACF) formation in female mice fed with high-fat diet. EGCG can suppress both PI3K/Akt and MAPK pathways that are crucial in obesity-associated colon cancer. EGCG also directly interacts with the PI3K downstream target bcl-2, an anti-apoptotic protein (Chen, 2012; Ermakova et al., 2005; Leone et al., 2003). In addition, EGCG can inhibit a transcriptional factor, NF- $\mathrm{BB}$, by another downstream target of the PI3K/Akt pathway (Ahmad et al., 2000). EGCG can also cause cell cycle arrest. EGCG reduced risk of cancer in obesity by increasing serum level of IGFBP-3, reducing the serum levels of IGF-I, leptin and insulin. Interestingly, EGCG can inhibit activated signal pathways of obesity-associated colon cancer at several points (Shimizu et al., 2008).

\section{Curcumin (Tumeric)}

Curcumin (Curcuma longa) is the major constituent of turmeric. It has been shown to prevent intestinal tumours in chemically induced tumours in rats and mice and decrease intestinal polyps in the APC Min mice (Jackson and Evers, 2009; Perkins et al., 2002). Clinically, some studies reported that dietary curcumin can reverse, stop, or suppress tumour progression in high-risk patients (Sharma et al., 2004). Turmeric component in curcumin has also been demonstrated to reduce obesity-induced polyp formation in animal models through inhibition of P13/Akt and $\beta$-catenin signal pathways (Ju et al., 2005; Shimizu et al., 2008). In another study, curcumin fed to APC Min mice with high fat diet intake, reduced the polyps number induced by high fat diet. APC Min mice fed with high fat diet (HFD) for 12 weeks had a $23 \%$ increase in total number of polyps compared to APC Min mice on the common rodent chow (RC). It was found that curcumin also enhanced and increased the anti-tumour effect of curcumin independent of high fat diet, as reported by other studies (Jackson and Evers, 2009, Mahmoud et al., 2000; Christina et al., 2011). At a cellular environment, curcumin was shown to cause growth arrest and DNA damage and induced 153 (GADD153) genes which apparently have a straight role in activating apoptosis (Scott et al., 2007). Initiation of apoptosis may be part of the mTOR signalling pathway as curcumin has been reported to suppress mTOR phosphorylation and its downstream effector target S6K1 throughout apoptosis (Beevers et al., 2009). Curcumin was shown to stop the increased body weight associated with a high fat diet. Body weight was notably higher in APC Min mice fed with HFD compared to mice fed with HFD plus curcumin (Christina et al., 2011).

\section{Polyphenols in Ginger and Honey}

Ginger or scientifically known as Zingiber officinale consists of phenolic compounds including shogaol, gingerol, paradol, and zerumbone that have been shown to have antioxidant, anti-tumour and anti-inflammatory properties (Beevers et al., 2009; Banerjee et al., 2011; Harliyansyah et al., 2007). Ginger extract and its bioactive constituents were demonstrated to suppress the growth of many types of cancers including colon cancer cells mainly by inhibiting proliferation, promoting apoptosis, arresting cells at Go/G1 cell cyle and inactivating NFkB activity (Khater, 2010; Habib et al., 2008; Abdullah et al., 2010). A study reported that supplementation for 28 days daily with ginger in 20 patients at high risk of colorectal cancer resulted in a decreased proliferation rate (decreased expression of Ki-67) but had lower effect on apoptosis in the normal appearing colonic mucosa cells crypts (Citronberg et al., 2013). Combination of ginger extract and Gelam honey which contain very high phenolic moieties were proven to have anti-cancer properties by inhibiting cell proliferation and causing DNA damage, cell cycle arrest and induction of apoptosis in colon cancer cells HT29. The chemopreventive effect of the combined treatment modulated Ras/ERK and P13/AKT pathway genes (Abdullah Tahir et al., 2015). In addition, Wen et al., (2012) showed that the combination between Gelam honey and ginger can act as a potential therapy in colorectal cancer treatment by suppression of mTOR, Wnt/ $\beta$ catenin signalling pathways and initiation of apoptosis pathway. On its own, Gelam honey induced apoptosis and supressed inflammation in colon cancer HT29 cells.

\section{Quercetin in Berries and Other Fruits}

Quercetin is a flavonol mostly found in many fruits and vegetables. The food sources of quercetin include tea, onions, apples, allium vegetables, berries, cucumber, sweet potato, cruciferous vegetables, beans, fruits and even some herbs such as sage, rosemary and oregano (Djurica et al., 2012). Experimental models of colon cancer suggest that quercetin can inhibit colon tumour formation in vivo and proliferation of colon tumour cells in vitro (Miyamoto et al., 2010; Gerhauser, 2008; Yang et al., 2000). Quercetin also reduced high fat diet-induced formation of aberrant crypt foci (Murakami et al., 2008). Site-specific preventive properties of quercetin in the colon unfortunately were not investigated, but quercetin did have some preventive activity in distal colon against 
chemically-induced tumours (Warren et al., 2009).

\section{Piper Betle}

P. betle is one of the Piperaceae families and is thought to originate from South East Asia (Abrahim et al., 2012). The leaves of P.betle contain a lot of bioactive compounds such as eugenol, chavicol, chavibetol and allypyrocatechol that have medicinal values including antioxidant, anticancer, antidiabetic and anti-infective properties (Kumar et al., 2010). It had been reported that some other medicinal properties of P.betle includes inhibition of platelet aggregation, antidiabetic activities, immunomodulatory properties and anti-allergic activities (Shen et al., 2007). Some studies have shown the anti-tumour effects of P.betle such as in oral, nasopharyngeal and breast cancer (Abrahim et al., 2012; Shun et al., 2007; Fathilah et al., 2009). For example, the leaves extract of P.betle demonstrated anti-tumour property in oral cancer by exhibiting anti-proliferative actions towards $\kappa \mathrm{B}$ cells (Abrahim et al., 2012). P.betle was also shown to have anti-cancer properties against nasopharyngeal epidermoid carcinoma cell line and breast cancer cell line (Fathilah et al., 2009, Widowati et al., 2011). It was also shown that P.betle inhibited colon carcinogenesis by downregulating both $\beta$-catenin and KRAS in the early phase of colon carcinogenesis (Widowati et al., 2011; Esa et al., 2013). AOM administration showed formation of ACF at 8 and 24 weeks. P.betle, however, did not reduce ACF formation at either week, but it managed to reduce beta-catenin expression and KRAS found highly expressed in the AOM group (Thirumalai et al., 2014).

In conclusion, there are many factors that lead to obesity-associated colon cancer and the mechanisms behind it, are still yet to be unravelled. Activation of certain genes that leads to abnormal levels of hormones, cytokines, growth factors have been shown to be associated with obesity associated colon cancer. Beneficial effects of vegetables and fruits in relation to cancer prevention have been proven in many studies. However, the metabolism and molecular biological properties of these phytochemical in chemoprevention still need to be further explored. Antioxidants-rich food can either be used alone or in combination in the prevention of not only colon cancer but also in other cancers as well. Can dietary modifications (low consumption of red meat, refined sugars and starches, sugars) and lifestyle changes (avoidance of alcohol, smoking and prevention of weight gain) be an answer to lowering the risk of colon cancer? Based on current and past evidences the answer is affirmative but perhaps a larger cohort study all around the world is still needed to address this issue.

\section{Acknowledgments}

The authors are grateful to Biochemistry Department of The National University of Malaysia for their unstinted support. This work was supported by Dana Impak Perdana Grant (DIP-2014-032).

\section{References}

Abdullah S, Abidin SAZ, Murad NA, et al (2010). Ginger extract (Zingiber officinale) triggers apoptosis and G0/G1 cells arrest in HCT 116 and HT 29 colon cancer cell lines. Afr J Biochem Res, 4, 134-42

Abdullah TA, Abdul Sani NF, Murad NA, et al (2005). Combined ginger extract and Gelam Honey Modulate Ras/ERKand P13K/AKT pathway genes in colon cancer HT29 cells. Nutr J, 14, 1-10.

Abrahim NN, Kanthimathi MS, Abdul Aziz A (2012). Piper Betle shows antioxidant activities, inhibits MCF-7 cell proliferation and increases activities of catalase and superoxide dismutase. BMC Complement Altern Med, 12, 220.

Aggarwal BB, Kunnumakkara AB, Harikumar KB, et al (2009). Signal transducer and activator of transcription-3, inflammation, and cancer: how intimate is the relationship?. Ann N Y Acad Sci, 1171, 59-76.

Aggarwal BB, Shishodia S (2006) Molecular targets of dietary agents for prevention and therapy of cancer. Biochem Pharmacol, 71, 1397-1421.

Ahmad N, Gupta S, Mukhtar H (2000). Green tea polyphenol epigallocatechin-3-gallate differentially modulates nuclear factor kappaB in cancer cells versus normal cells. Arch Biochem Biophys, 376, 338-46.

Arends JW (2000). Molecular interactions in the Vogelstein model of colorectal carcinoma. J Pathol, 190, 412-16.

Baba Y, Nosho K, Shima K, et al (2010). Prognostic significance of AMP-activated protein kinase expression and modifying effect of MAPK3/1 in colorectal cancer. Br J Cancer, 103, 1025-33.

Banerjee S, Mullick H, Banerjee J, Ghosh A (2011). Zingiber officinale:'a natural gold'. Int $J$ Pharmaceutical Bio-Sci, 2, 283-94.

Beevers CS, Chen L, Liu L, et al (2009). Curcumin disrupts the mammalian target of rapamycin-raptor complex. Cancer Res, 69, 1000-8.

Ben Q, An W, Jiang Y, et al (2012). Body mass index increases risk for colorectal adenomas based on meta-analysis. Gastroenterology, 142, 762-72.

Boyle P, Langman JS (2000). ABC of colorectal cancer: Epidemiology. BMJ, 32, 805-8.

Calle EE., Rodriguez C, Walker-Thurmond K, Thun MJ (2003). Overweight, obesity, and mortality from cancer in a prospectively studied cohort of U.S. Adults. $N$ Engl $J$ Med, 348, 1625-38.

Cecil JE, Tavendale R, Watt P, et al (2008). An obesity-associated FTO gene variant and increased energy intake in children. $N$ Engl J Med, 359, 2558-66.

Chen J (2011). Targeted therapy of obesity-associated colon cancer. Translational Gastrointestinal Cancer, 1, 44-57.

Chen J (2012). Prevention of obesity-associated colon cancer by (-)-epigallocatechin-3-gallate and curcumin. Translation Gastrointestinal Cancer, 1, 243-9.

Chen J, Yang J, and Zhao KN (2013). FTO gene, obesity and colon cancer from epidemiological evidence to laboratory studies. Translation Gastrointestinal Cancer, 2, 194-203.

Christina PB, John M, Ruby M, Warren L (2011). Curcumin suppresses intestinal polyps in APC Min mice fed a high fat diet. Pathobiol Aging Age Relat Dis, 1, 1.

Citronberg J, Bostick R, Ahearn T, et al (2013). Effects of ginger supplementation on cell-cycle biomarkers in the normal-appearing colonic mucosa of patients at increased risk for colorectal cancer: results from a pilot, randomized, and controlled trial. Cancer Prev Res (Phila), 6, 271-81.

Davies RW, Lau P, Naing T, et al (2013). A 680 kb duplication at the FTO locus in a kindred with obesity and a distinct body 
fat distribution. Eur J Hum Genet, 21, 1417-22.

de Jong AE, Morreau H, Nagengast FM, et al (2005). Prevalence of adenomas among young individuals at average risk for colorectal cancer. Am J Gastroenterol, 100, 139-143.

Djurica Z, Seversonb RK, Kato I (2012). Association of dietary quercetin with reduced risk of proximal colon cancer. Nutr Cancer, 64, 351-60.

Dora C, Giovanni B, Franco B, Luigi R (2013). Molecular pathways involved in colorectal cancer: Implications for disease behavior and prevention. Int J Mol Sci, 14, 16365-85.

Dougkas A, Yaqoob P, Givens DI, et al (2013). The impact of obesity-related SNP on appetite and energy intake. $\mathrm{Br} J$ Nutr, 110, 1151-6.

Drew JE (2012). Moleular mechanism linking adipokines to obesity-related colon cancer: focus on leptin. Proc Nutr Soc, 71, 175-80.

Du GJ, Wang CZ, Qi LW, et al (2012). The synergistic apoptotic interaction of panaxadiol and epigallocatechin gallate in human colorectal cancer cells. Phytother, 27, 272-7.

el-Deiry WS, Tokino T, Velculescu VE, et al (1993). WAF1, a potential mediator of p53 tumor suppression. Cell, 75, 817-25.

Ermakova S, Choi BY, Choi HS, et al (2005). The intermediate filament protein vimentin is a new target for epigallocatechin gallate. J Biol Chem, 280, 16882-90.

Esa F, Wan Ngah WZ, A.Rahman AJ, Mohd Yusof YA (2013). Inhibition of b-catenin and KRAS expressions by piper betle in azoxymethane-induced colon cancer of male fischer 344 rats. Anal Quant Cytol Histol, 35, 324-31.

Fathilah AR, Yusoff M, Rahim ZHA (2009). The effect of Psidiumguajava and Piper betle extracts on the morphology of dental plaque bacteria. Pak J Med Sci, 25, 926-33.

Fenton JI, Birmingham JM (2010). Adipokine regulation of colon cancer: adiponectin attenuates interleukin-6-induced colon carcinoma cell proliferation via STAT-3. Mol Carcinog, 49, 700- 09 .

Fenton JI, Birmingham JM, Hursting SD, NG H (2008). Adiponectin blocks multiple signalling cascades associated with leptin-induced cell proliferation in Apc Min/+ colon epithelial cells. Int J Cancer, 122, 2437-45.

Fierz Y, Novosyadlyy R, Vijayakumar A, Yakar S, LeRoith D (2010). Mammalian target of rapamycin inhibition abrogates insulin mediated mammary tumor progression in type 2 diabetes. Endocr Relat Cancer, 17, 941-51.

Flores MB, Rocha GZ, Damas-Souza DM, et al (2012). Obesity-induced increase in tumor necrosis factor- $\alpha$ leads to development of colon cancer in mice. Gastroenterology, 143, 741-53.

Gerhauser C (2008). Cancer chemopreventive potential of apples, apple juice, and apple components, Planta Med, 74, 1608-24.

Gislette T, Chen J (2010). The possible role of IL-17 in obesity-associated cancer. Sci World J, 17, 785-790.

Grande M, Milito G, Attinà GM, et al (2008). Evaluation of clinical, laboratory and morphologic prognostic factors in colon cancer. World J Surg Oncol, 98, 1-7.

Guerrero S, Casanova I, Farre L, et al (2000). K-ras codon 12 mutation induces higher level of resistance to apoptosis and predisposition to anchorage-independent growth than codon 13 mutation or proto-oncogene overexpression. Cancer Res, 60, 6750-6.

Habib SH, Makpol S, Abdul Hamid NA, et al (2008). Ginger extract (Zingiber officinale) has anti-cancer and anti-inflammatory effects on ethionine-induced hepatoma rats. Clinics (Sao Paulo), 63, 807-13.

Haggar FA, Boushey RP (2009). Colorectal cancer epidemiology: incidence, mortality, survival, and risk factors. Clin Colon
Rectal Surg, 22, 191-7.

Harliyansyah H, Murad NA, Wan Ngah WZ, et al (2007). Antiproliferative, antioxidant and apoptosis effects of Zingiber officinale and 6-gingerol on HepG2 cells. Asian J Biochem, 2, 421-6.

Horst D, Chen J, Morikawa T, et al (2012). Differential WNT activity in colorectal cancer confers limited tumorigenic potential and is regulated by MAPK signalling. Cancer Res, 72, 1547-56.

Jackson LN, Evers BM (2009). Regulation of proliferation, apoptosis and cell cycle in gastrointestinal disorders. Curr Opin Pharmacol, 9, 708-14.

Jaffe T, Schwartz B (2008). Leptin promotes motility and invasiveness in human colon cancer cells by activating multiple signal transduction pathways. Int $J$ Cancer, 123, 2543-56.

Janice ED (2014). Signalling pathways implicated in obesity associated cancers. Open Obes $J$, 6, 44-49.

Janout V, Kollárová H (2001). Epidemiology of colorectal cancer. Biomed Pap Med Fac Univ Palacku Olomouc Czech Repub, 145, 5-10.

Ju J, Hong J, Zhou JN, et al (2005). Inhibition of intestinal tumorigenesis in Apcmin/+ mice by (-)-epigallocatechin3 -gallate, the major catechin in green tea. Cancer Res, $\mathbf{6 5}$, 10623-31.

Khandekar MJ, Cohen P, Spielgelman BM (2011). Molecular mechnisms of cancer development in obesity. Nat Rev Cancer, 11, 886-95.

Kumar N, Misra P, Dube A(2010). Piper Betle Linn. A maglinant Pan-Asiatic plant with an array of pharmacological activities and prospects for drug discovery. Curr Sci, 99, 922-32.

Labayen I, Ruiz JR, Ortega FB, et al (2011). Association between the FTO rs9939609 polymorphism and leptin in European adolescents: a possible link with energy balance control. The HELENA study. Int J Obes (Lond), 35, 66-71.

Leary RJ, Lin JC, Cummins J, et al (2008). Integrated analysis of homozygous deletions, focal amplifications, and sequence alterations in breast and colorectal cancers. Proc Natl Acad Sci US A, 105, 16224-29.

Leone M, Zhai D, Sareth S, et al (2003). Cancer prevention by tea polyphenols is linked to their direct inhibition of antiapoptotic Bcl-2-family proteins. Cancer Res, 63, 8118-21.

Lin JK, Chang SC, Yang YC, Li AF (2003). Loss of heterozygosity and DNA aneuploidy in colorectal adenocarcinoma. Ann Surg Oncol, 10, 1086-94.

Lo CW, Chen MW, Hsiao M, et al (2010). IL-6 trans-signaling in formation and progression of malignant ascites in ovarian cancer. Cancer Res, 71, 424-34.

Mak JC (2012). Potential role of green tea catechins in various disease therapies: Progress and promise. Clin Exp Pharmacol Physiol, 39, 265-73.

Malumbres M, Barbacid M (2003). RAS oncogenes: The first 30 years. Nat Rev Cancer, 3, 459-65.

Miyamoto S, Yasui Y, Ohigashi H, Tanaka T, Murakami A(2010). Dietary flavonoids suppress azoxymethane-induced colonic preneoplastic lesions in male C57BL/KsJ-db/db mice. Chem Biol Interact, 183, 276-83.

Monghaddam AA, Woodward M, Huxley R (2007). Obesity and risk of colorectal Cancer. Ameta-analysis. Cancer Epidemiol Biomarkers Prev, 16, 2533-47.

Murakami A, Ashida H, Terao J (2008). Multitargeted cancer prevention by quercetin, Cancer Lett, 269, 315-25.

Nakazato T, Ito K, Ikeda Y, Kizaki M (2005). Green tea component, catechin, induces apoptosis of human malignant B cells via production of reactive oxygen species. Clin Cancer Res, 11, 6040-49. 
National Cancer Registry, Ministry of Health Malaysia (2006). Malaysia Cancer Statistic - Data and Figure Peninsular Malaysia 2006.

National Center for Health Statistics. Health, United States, 2015: With Special Feature on Racial Exit Disclaimer and Ethnic Health Disparities Exit Disclaimer. Hyattsville, MD. 2016.

National Health and Morbidity Survey. (2006). Ministry of Health, Malaysia. Institute for Public Health, Kuala Lumpur 2006.

Novellasdemunt L, Antas P, Li VS (2015). Targeting Wnt signalling in colorectal cancer. A Review in the Theme: Cell Signalling: Proteins, Pathways and Mechanisms. Am J Physiol Cell Physiol, 309, 511-21.

Novosyadlyy R, Lann DE, Vijayakumar A, et al (2010). Insulin-mediated acceleration of breast cancer development and progression in a non-obese model of type 2 diabetes. Cancer Res, 70, 741-51.

Nunez NP, Hursting SD, Yakar S, Fowler D, Vinson C (2009). Obesity provides a permissive milieu in inflammationassociated carcinogenesis: analysis of insulin and IGF pathways. Methods Mol Biol, 512, 29-37.

Ogino S, Odze RD, Kawasaki T, et al (2006). Correlation of pathologic features with $\mathrm{CpG}$ island methylator phenotype (CIMP) by quantitative DNA methylation analysis in colorectal carcinoma. Am J Surg Pathol, 30, 1175-83.

Oikawa T, Okuda M, Ma Z, et al (2005). Transcriptional control of BubR1 by p53 and suppression of centrosome amplification by BubR1. Mol Cell Biol, 25, 4046-61.

Olivo-Marston SE, Hursting SD, Lavigne J, et al (2009). Genetic reduction of circulating insulin-like growth factor- 1 inhibits azoxymethane-induced colon tumorigenesis in mice. $\mathrm{Mol}$ Carcinog, 48, 1071-76.

Olszewski PK, Fredriksson R, Olszewska AM, et al (2009). Hypothalamic FTO is associated with the regulation of energy intake not feeding reward. BMC Neuroscience, 10, $1-12$.

Orner GA, Dashwood WM, Blum CA, et al (2002). Suppression of tumorigenesis in the $\mathrm{Apc}(\mathrm{min})$ mouse: down-regulation of beta-catenin signaling by a combination of tea plus sulindac, Carcinogenesis, 24, 263-7.

Percik R, Stumvoll M (2009). Obesity and cancer. Exp Clin Endocrinol Diabetes, 117, 563 - 66.

Perkins S, Verschoyle RD, Hill K, et al (2002). Chemopreventive efficacy and pharmacokinetics of curcumin in the $\mathrm{min} /+$ mouse, a model of familial adenomatous polyposis. Cancer Epidemiol Biomarkers Prev, 11, 535-40.

Pino MS, Chung DC (2010). The chromosomal instability pathway in colon cancer. Gastroenterology, 138, 2059-72.

Pischon T, Lahmann PH, Boeing H, et al (2006). Body Size and Risk of Colon and Rectal Cancer in the European Prospective Investigation Into Cancer and Nutrition (EPIC). JNCI J Natl Cancer Inst, 98, 920-31.

Pitman RT, Fong JT, Billman P, Puri N (2012). Knockdown of the fat mass and obesity gene disrupts cellular energy balance in a cell-type specific manner. PLoS One, 7, e38444.

Riondino S, Roselli M, Palmirotta R, et al (2014). Obesity and colorectal cancer: Role of adipokines in tumor initiation and progression. World J Gastroenterol, 20, 5177-90.

Sameer SA (2013). Colorectal cancer: Molecular mutations and polymorphisms. Frontiers in oncology. Cancer Epidemiol Prev, 3, 1-8.

Samowitz WS, Albertsen H, Herrick J, et al (2005). Evaluation of a large, population-based sample supports a $\mathrm{CpG}$ island methylator phenotype in colon cancer. Gastroenterology, 129, 837-45.

Saxena NK, Fu PP, Nagalingam A, et al (2010). Adiponectin modulates $\mathrm{C}$-jun $\mathrm{N}$-terminal kinase and mammalian target of rapamycin and inhibits hepatocellular carcinoma. Gastroenterology, 139, 1762-73.

Scott DW, Loo G (2007). Curcumin induced GADD153 upregulation: modulation by glutathione. $J$ Cell Biochem, 101, 307-20.

Sharma RA, Euden SA, Platton SL, et al (2004). Phase I clinical trial of oral curcumin: biomarkers of systemic activity and compliance. J Am Assoc Cancer Res, 10, 6847-54.

Shih IM, Zhou W, Goodman SN, et al (2001). Evidence that genetic instability occurs at an early stage of colorectal tumorigenesis. Cancer Res, 61, 818-22.

Shimizu M, Shirakami Y, Sakai H (2008). (-)-epigallocatechin Gallate suppresses Axozymethane-induced colonic premalignant lesions in male $\mathrm{C} 57 \mathrm{BL} / \mathrm{KsJ}-\mathrm{db} / \mathrm{db}$ mice. Cancer Prev Res (Phila), 1, 298-304.

Shun CY, Chau JW, Jing JL, et al (2007). Protection effect of Piper betle leaf extract against carbon tetrachloride-induced liver fibrosis in rats. Arch Toxicol, 81, 45-55.

Sieber OM, Lamlum H, Crabtree MD, et al (2012). Whole-gene APC deletions cause classical familial adenomatous polyposis, but not attenuated polyposis or "multiple" colorectal adenomas. Proc Natl Acad Sci U S A, 99, 2954-58.

Sikilidis AK, VAramini B (2001). Roles of hormone and signalling molecules in describing the relationship between obesity and colon cancer. Pathol Oncol Res, 17, 785-90.

Sonestedt E, Gullberg B, Ericson U, et al (2011). Association between fat intake, physical activity and mortality depending on genetic variation in FTO. Int J Obes (Lond), 35, 1041-49.

Speakman JR, Rance KA, Johnstone AM (2008). Polymorphisms of the FTO gene are associated with variation in energy intake, but not energy expenditure. Obesity (Silver Spring), 16, 1961-65.

Suganuma M, Ohkura Y, Okabe S, et al (2001). Combination cancer chemoprevention with green tea extract and sulindac shown in intestinal tumor formation in Min mice. J Cancer Res Clin Oncol, 127, 69-72.

Sunkara R, Verghese M, Walker LT, Shackelford L (2009). Suppression of colon cancer development in an Azoxymethane-Fisher 344 rat model by cranberry. Res $J$ Phytochem, 3, 25-34.

Swamy MV, Herzog CR, Rao CV (2003). Inhibition of COX-2 in colon cancer cell lines by celecoxib increases the nuclear localization of active p53. Cancer Res, 63, 5239-42.

The Star. (2014, June 16). Malaysia's obesity rate highest in Asia. Retrieved October 3, 2014, from http://www.thestar.com. my/News/Nation/2014/06/16/obesity-malaysia-highest-inasiasays-pm-science-advisor/.

Timpson NJ, Emmett PM, Frayling TM, et al (2008). The fat mass-and obesity-associated locus and dietary intake in children. Am J Clin Nutr, 88, 971-8.

Van de Flier LG, Sabates-Beller J, Oving I, et al (2007). The intestinal Wnt/TCF signature. Gastrpenterology, 132, 628-32.

Waldner MJ, Foersch S, Neurath MF (2012). Interleukin-6--a key regulator of colorectal cancer development. Int J Biol Sci, 8, 1248-53.

Warren CA, Paulhill KJ, Davidson LA, et al (2009). Quercetin may suppress rat aberrant crypt foci formation by suppressing inflammatory mediators that influence proliferation and apoptosis. J Nutr, 139, 101-5.

Wen CTP, Hussein SZ, Abdullah S, et al (2012). Gelam and nenas honeys inhibit proliferation of HT 29 colon cancer cells by inducing DNA damage and apoptosis while suppressing inflammation. Asian Pac J Cancer Prev, 13, 1605-10.

WHO (2015). Obesity and overweight. Fact sheet $\mathrm{N}^{\circ} 311$. World Health Organization, Geneva; 2015. 
Widowati W, Mozef T, Risdian C, et al (2011). The comparison of antioxidative and proliferation inhibitor properties of Piper betle L, Catharanthus roseus [L] G.Don, Dendrophtoe petandra L., Curcuma mangga Val. Extracts on T47D Cancer Cell Line. Int J Biosci Biochem Bioinforma, 1, 22-8.

Wollin KY, Carson K, Colditz GA (2010). Obesity and cancer. Oncologist, 15, 556-65.

Yamamoto K, Rose-John S (2012). Therapeutic blockade of interleukin-6 in chronic inflammatory disease. Clin Pharmacol Ther, 91, 574-6.

Yang Jian (2012). FTO genotype is associated with phenotypic variability of body mass index. Nature, 490, 267-72.

Yang K, Lamprecht SA, Liu Y, Shinozaki H, Fan K (2000). Chemoprevention studies of the flavonoids quercetin and rutin in normal and azoxymethane-treated mouse colon. Carcinogenesis, 21, 1655-60.

Yu H, Pardoll D, Jove R (2009). STATs in cancer inflammation and immunity: a leading role for STAT3. Nat Rev Cancer, 9, 798-809.

Zhang X, Qi Q, Zhang C, et al (2012). FTO Genotype and 2-year Change in Body Composition and Fat Distribution in Response to weight-loss Diets: The POUNDS LOST Trial. Diabetes, 61, 3005-11.

Zisman AL, Nickolov A, Brand RE, Gorchow A, Roy HK (2006). Associations between the age at diagnosis and location of colorectal cancer and the use of alcohol and tobacco: implications for screening. Arch Intern Med, 166, 629-34.

\section{c) (7) (8)}

This work is licensed under a Creative Commons AttributionNon Commercial 4.0 International License. 